\title{
BMJ Open Prevalence and determinants of current and secondhand smoking in Greece: results from the Global Adult Tobacco Survey (GATS) study
}

George Rachiotis, ${ }^{1}$ Anastasia Barbouni, ${ }^{2}$ Antonis Katsioulis, ${ }^{1}$ Eleni Antoniadou, ${ }^{2}$ Konstantinos Kostikas, ${ }^{1}$ Kyriakoula Merakou, ${ }^{2}$ Kallirrhoe Kourea, ${ }^{1}$ Rula N Khoury, ${ }^{3}$ Agis Tsouros, ${ }^{3}$ Jenny Kremastinou, ${ }^{2}$ Christos Hadjichristodoulou ${ }^{1}$

To cite: Rachiotis G, Barbouni A, Katsioulis A, et al. Prevalence and determinants of current and secondhand smoking in Greece: results from the Global Adult Tobacco Survey (GATS) study. BMJ Open 2017;7:e013150.

doi:10.1136/bmjopen-2016013150

- Prepublication history for this paper is available online. To view these files please visit the journal online (http://dx.doi.org/10.1136/ bmjopen-2016-013150).

GR and $A B$ contributed equally as first authors.

Received 30 June 2016 Revised 30 August 2016 Accepted 28 October 2016

CrossMark

For numbered affiliations see end of article.

Correspondence to Dr Christos Hadjichristodoulou; xhatzi@med.uth.gr

\section{ABSTRACT}

Objectives: Greece is one of the leading tobaccoproducing countries in European Union, and every year over 19000 Greeks die from tobacco-attributable diseases. The aim of the present study was to provide nationally representative estimates on current and secondhand smoking prevalence in Greece and their determinants.

Design: Cross-sectional.

Setting: Greece.

Participants: A total of 4359 individuals participated in the Global Adult Tobacco Survey (GATS), a household survey of adults $\geq 15$ years old (overall response rate $69 \%$ ). They were selected through a multistage geographically clustered sampling design with face-to-face interview.

Primary and secondary outcome measures: In 2013, we investigated the prevalence of current and secondhand smoking and their determinants.

Univariate and logistic regression analysis was used in order to identify possible risk factors associated with the prevalence of current and secondhand smoking.

Results: The prevalence of current smoking was $38.2 \%$ (95\% Cl $35.7 \%$ to $40.8 \%$ ), and the mean number of cigarettes smoked per day was 19.8 . Multivariate analysis confirmed that male gender (OR=3.24; $95 \% \mathrm{Cl} 2.62$ to 4.00 ), age groups (25-39, $\mathrm{OR}=4.49 ; 95 \% \mathrm{Cl} 3.09$ to 8.46 and $40-54, \mathrm{OR}=3.51$; $95 \% \mathrm{Cl} 1.88$ to 5.87$)$ and high school education (OR=1.97; $95 \% \mathrm{Cl} 1.41$ to 2.74 ) were independently associated with the current smoking. Remarkably, responders with primary or less education had the lowest prevalence of current smoking $(p<0.001)$. The prevalence of exposure to secondhand smoke at work, home and restaurants, was $52.3 \%, 65.7 \%$ and $72.2 \%$. In total, $90.0 \%$ (95\% Cl $87.8 \%$ to $91.9 \%$ ) of Greek population is exposed to tobacco smoke (current smoking and secondhand smoke).

Conclusions: Our results revealed an extremely high prevalence of current smoking and exposure to secondhand smoke among the adult population and a positive gradient between education and current smoking. These findings are alarming and implementation of comprehensive tobacco control and

\section{Strengths and limitations of this study}

- Nationwide, representative sample of Greek adult population.

- Global WHO standardised methodology to ensure comparability across countries: standardised questionnaire and sampling framework.

- The questionnaires were collected through interviews resulting in satisfactory response rate and limited missing values.

- The main limitation of our study is related to the self-reported nature of the data selected.

prevention strategies could be impactful in fighting the tobacco epidemic in Greece.

\section{INTRODUCTION}

Tobacco use is a major contributor to mortality and an estimated $9 \%$ of the global deaths are attributable to exposure to tobacco while in high income about $18 \%$ of deaths are attributable to tobacco. ${ }^{12}$ Projected estimates from low-income and middle-income countries suggest that tobacco exposure will cause 1000 billion premature deaths during 21st century. $^{2}$ Greece is a leading tobacco producing country in the context of the European Union. ${ }^{3}$ It has been estimated that every year in Greece more than 19000 Greeks die from tobacco-attributable diseases (cancers, cardiovascular and diseases of the respiratory system). Moreover, in 2011, tobacco exposure accounted for almost $9 \%$ of the national total of hospital admissions. Moreover, it has been estimated that smoking is associated with 284498 years of potential life lost per year among adults (all sexes) 35-65 years old. ${ }^{4}$ There is some evidence that Greece 
presents a high prevalence of smoking among adults. ${ }^{5}$ In addition, previous cross-sectional studies (general population or workplace based) demonstrated a high prevalence of smoking and high prevalence of the use of smoking across several socioeconomic and demographic groups. ${ }^{6-11} \mathrm{~A}$ set of six strategies (MPOWER) has been recommended by the WHO: Monitoring tobacco use and Prevention policies; Protecting people from tobacco smoke; Offering help to quit tobacco use; Warning about the dangers of tobacco; Enforcing bans on tobacco advertising, promotion and sponsorship; and Raising taxes on tobacco. ${ }^{12}$ Monitoring of the tobacco epidemic is key to effectively managing it. Many countries conduct surveys aiming to monitor adult tobacco use, but no one standard global survey for adults has tracked tobacco use, exposure to secondhand tobacco smoke and tobacco control measures. The Global Adult Tobacco Survey (GATS) fills the need for standardised survey of tobacco use. In particular, GATS is a nationally representative survey that uses a standardised protocol, to ensure comparability across countries, to track tobacco use and evaluate tobacco control policies. ${ }^{13} 14$ GATS has been implemented worldwide since 2008. The aims of this study are to examine the prevalence and determinants of current smoking and exposure to secondhand smoke among Greek adults.

\section{METHODS}

We used data from the 2013 Greece GATS, a household survey of adults ( $\geq 15$ years old) aiming to produce nationally representative data, a multicentre cluster sample design is used in all countries.

The household and individual questionnaires were based on the GATS Core Questionnaire with optional questions, which were designed for use in countries implementing GATS. The individual questionnaire used included seven sections: background information, tobacco smoking, mode of tobacco use in any way other than smoking, cessation, secondhand smoke, economics, media, knowledge, attitudes and practices. The use of household questionnaire aimed to collect information on all adult residents-based on sampling strategy-in the household in order to randomly select an eligible respondent to complete the individual questionnaire. These questionnaires (household and individual) were adapted and modified to reflect issues relevant and applicable to the situation in Greece. Additional questions were included in the questionnaire on the use of electronic cigarette and the support of the law prohibiting smoking in public places. The questionnaire was first developed in English then translated into Greek and later was also back translated from Greek into English and was finalised in June 2013. ${ }^{15}$ Current smokers were considered persons who currently smoke tobacco. Current use includes both daily and occasional use. Secondhand smoking referred to smoking exposure at home and (during the past 30 days) in public places (the work place, government buildings/offices, healthcare facilities, restaurants, bars/night clubs, cafes/cafeterias, public transportation, schools, colleges/ universities and private workplaces). In Greece, GATS was conducted by the National School of Public Health, the Department of Hygiene and Epidemiology of the University of Thessaly, and the MRB Hellas, Market Research Company. The survey had the support of the Regional Office for Europe of the WHO and the Centers for Disease Control and Prevention (CDC) provided technical assistance in the design and implementation of the study.

\section{Greece GATS sampling methodology}

The sampling frame used for the GATS survey in Greece was created on the basis of the 2001 and 2011 Greek population census given that 2011 census provided only total figures. Data on gender, age and urban population allocation have been projected taking into account the 2001 census given the absence of availability of complete sampling frames at household level, stratified probability proportional to size (PPS) with replacement sampling has been applied in four stages. The strata apply to four major divisions of the Greek territory (Attica, Northern Greece, Central Greece, Crete and Aegean Islands). The principal sampling unit (PSU) was the Kapodistrian municipality $(n=1034)$. At the first stage of the sampling design, 76 discrete PSUs have been selected, whereas some of them more than once resulting in overall 100 PSU selections (due to the replacement approach). At the second stage, two segments (census sectors) have been selected for each PSU, while for those PSUs selected $\mathrm{K}$ times, the number of selected segments was equal to $2 \times \mathrm{K}$. In particular, for urban areas, the census sectors consist of nearby blocks of buildings, created in such way to account for 500 households each. For the rural areas, the census sectors were groups of nearby villages. As the census sectors size in terms of household number varies significantly, they have been selected with systematic PPS. For those PSU consisting of urban/rural census sectors the sampling method selected was the stratified systematic PPS where we defined the urbanicity of the PSU. Overall, 200 census sectors were selected. Following the standard protocol and recommendations outlined in the GATS Sample Design Manual, the initial target was a representative sample of 4000 households, subject to applicable non-response and eligibility rates. ${ }^{14}{ }^{15}$ After accounting for possible non-response and illegibility, an average of 33 households per selected census sector were randomly selected, resulting in a total sample size of 6600 households. ${ }^{16}{ }^{17}$ A total of 4359 individuals participated in the study (overall response rate $69.0 \%)$.

\section{Statistical analysis}

Complex survey data analysis was performed to obtain population estimates and their 95\% CIs. Sample weights were developed for each respondent following the 
standard procedures established in the GATS Sample Design and Sample Weights manuals. ${ }^{14}{ }^{15}$ The final weights were used in all analyses to produce population estimates and their CIs. In univariate analysis, Pearson's $\chi^{2}$ test was used to analyse associations between sociodemographic factors and current and secondhand smoking. Relative risk and the corresponding 95\% CIs were also calculated. In multivariate analysis, logistic regression analysis was performed to identify independent risk factors for the current and secondhand smoking. ORs and 95\% CIs were calculated. p Values were considered statistically significant if $p<0.05$. All weighting computations and all computations of estimates and their CIs were performed using the complex sample module of SPSS V.19.

\section{RESULTS}

Overall, $38.2 \%$ of the respondents were current tobacco smokers and among them, $36.6 \%$ were daily smokers (table 1). The mean number of cigarettes among daily smokers per day was 19.8 . The percentage of former smokers was estimated at $14.8 \%$. The current smoking and daily smoking prevalence for men and women were $51.2 \%, 49.7 \%$, and $25.7 \%$ and $23.9 \%$, respectively.

Regarding the age at daily smoking initiation among ever daily smokers was 20-34 years and it is remarkable that $52.2 \%$ of the ever daily smokers reported start smoking at age 17-19 years (results not shown). In addition, $25.5 \%$ of ever daily smokers reported smoking initiation at age $15-16$ years. Finally, $4.8 \%$ of ever daily smokers 20-34 years old reported smoking initiation at age $<15$ years.

Table 2 presents information on the prevalence of exposure to secondhand smoke. The prevalence of exposure to secondhand smoke at work, home and restaurants was $52.3 \%, 65.7 \%$ and $72.2 \%$, respectively. Exposure to secondhand smoke was high at bars/night clubs (95.8\%) and coffee shops (85.0\%). In addition, $43.6 \%$ of the persons who visited universities during the past 30 days were exposed to secondhand smoke.
Table 2 Prevalence of exposure to secondhand smoke at work, home and various public places

\begin{tabular}{lll}
\hline Secondhand exposure & $\begin{array}{l}\text { Overall } \\
(\%)\end{array}$ & $\begin{array}{l}\text { Non-smokers } \\
(\%)\end{array}$ \\
\hline At work & 52.3 & 40.0 \\
At home & 65.7 & 51.1 \\
At public places & & \\
$\quad$ Restaurants & 72.2 & 67.1 \\
Cafes, coffee shops, tea & 85.0 & 81.9 \\
houses & & \\
$\quad$ Bars, night clubs & 95.8 & 94.3 \\
$\quad$ Governmental buildings & 18.2 & 15.2 \\
$\quad$ Private workplaces & 55.6 & 45.7 \\
$\quad$ Universities & 43.6 & 41.3 \\
Schools & 9.2 & 8.1 \\
Public transportation & 6.9 & 5.6 \\
In favour of banning smoking in workplace & \\
$\quad$ Yes & 73.9 & 90.9 \\
In favour of banning smoking in public places \\
Yes \\
\multicolumn{2}{c}{61.4} \\
\hline \multicolumn{2}{c}{84.1} \\
\hline
\end{tabular}

Overall, over $60.0 \%$ of participants supported banning smoking in workplaces and public places, and among non-smokers the support was over $80.0 \%$.

In total, $90.0 \%$ (95\% CI $87.8 \%$ to $91.9 \%)$ of the Greek population is exposed to tobacco smoke (current smoking and secondhand smoke).

\section{Univariate and multivariate analysis of risk factors for current smoking}

Univariate analysis shows (table 3 ) that the sex ratio (man/woman) for smoking is $1.99(\mathrm{p}<0.001)$. The agespecific pattern of current smoking indicates an increased prevalence of smoking during young adulthood and declined after the age of 40 years $(p<0.001)$. With respect to educational status participants with primary or less education had the lowest prevalence of current smoking. There were no significant differences in smoking in terms of residential status (rural/urban).

Multivariate analysis (table 4) indicated that gender, age and education were independently associated with

Table 1 Percentage of adults ( $\geq 15$ years old), by detailed smoking status and gender-GATS Greece, 2013

\begin{tabular}{llcc}
\hline Smoking status & $\begin{array}{l}\text { Overall } \\
\text { Percentage }(95 \% \text { Cl) }\end{array}$ & Male & Female \\
\hline Current tobacco smoker & $38.2(35.7$ to 40.8$)$ & $51.2(47.9$ to 54.4$)$ & $25.7(22.7$ to 28.9$)$ \\
$\quad$ Daily smoker & $36.6(34.1$ to 39.1$)$ & $49.7(46.4$ to 53.0$)$ & $23.9(21.2$ to 26.9$)$ \\
Occasional smoker & $1.6(1.0$ to 2.6$)$ & $1.5(0.7$ to 2.9$)$ & $1.8(1.1$ to 2.9$)$ \\
Occasional smoker, formerly daily & $0.6(0.3$ to 1.2$)$ & $0.8(0.4$ to 1.6$)$ & $0.4(0.2$ to 1.0$)$ \\
Non-current smoker & $61.8(59.2$ to 64.3$)$ & $48.8(45.6$ to 52.1$)$ & $74.3(71.1$ to 77.3$)$ \\
$\quad$ Former smoker & $14.8(12.9$ to 16.9$)$ & $18.1(15.9$ to 20.6$)$ & $11.5(9.1$ to 14.5$)$ \\
Former daily smoker & $11.8(10.4$ to 13.3$)$ & $16.4(14.3$ to 18.7$)$ & $7.3(5.9$ to 9.0$)$ \\
Never daily smoker & $50.0(46.9$ to 53.1$)$ & $32.5(29.0$ to 36.2$)$ & $67.0(63.3$ to 70.6$)$ \\
Former occasional smoker & $3.0(2.1$ to 4.3$)$ & $1.8(1.1$ to 2.8$)$ & $4.2(2.7$ to 6.4$)$ \\
\hline
\end{tabular}

Current use includes both daily and occasional (less than daily) use.

GATS, Global Adult Tobacco Survey. 
Table 3 Univariate analysis of smoking prevalence and SHS (GATS, Greece, 2013)

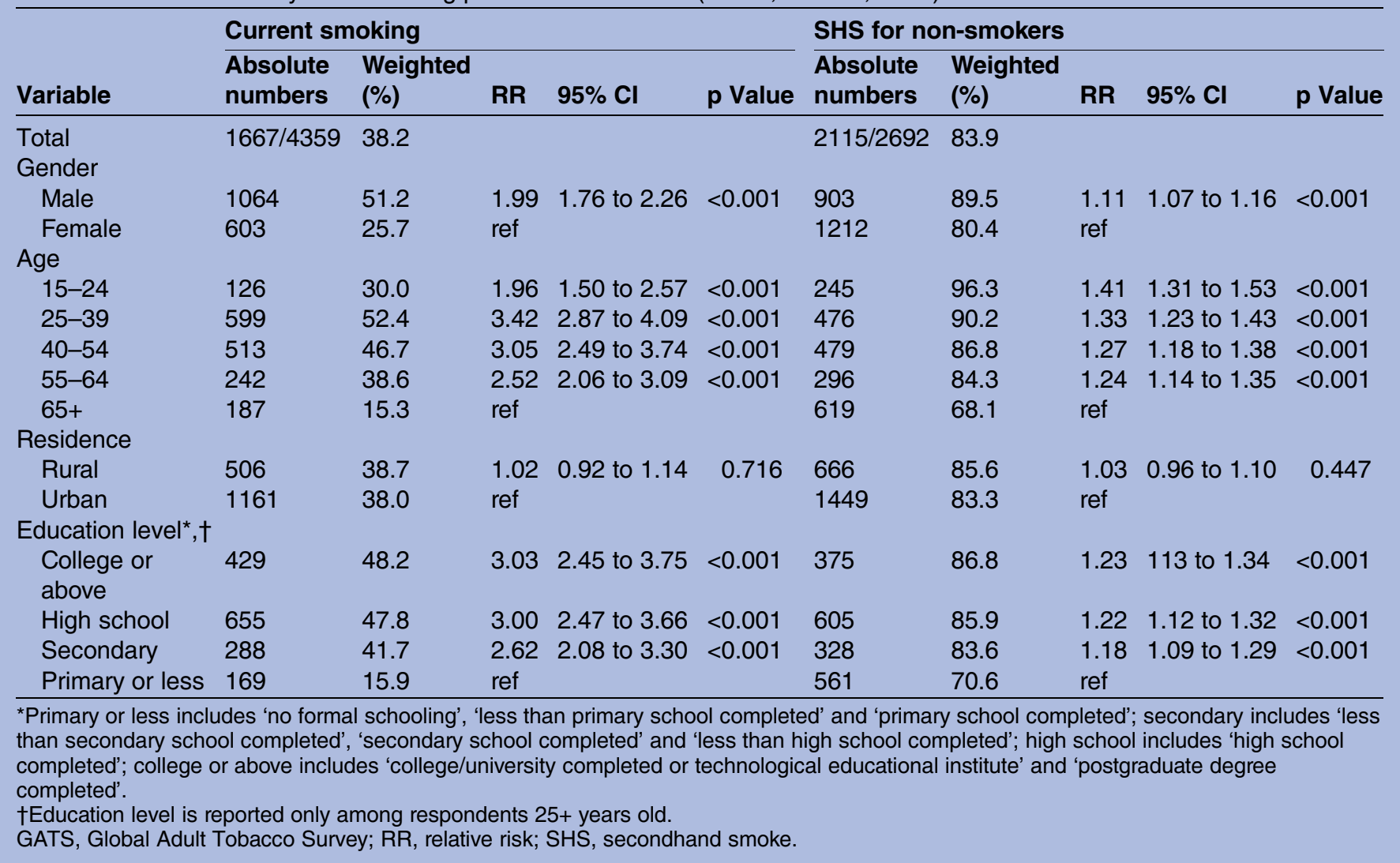

the current smoking. In particular, men were 3.24 times $(\mathrm{OR}=3.24 ; 95 \%$ CI 2.62 to 4.00$)$ more likely to report current smoking compared with women. Current smoking declined with increasing age. Multivariate analysis showed a complex smoking pattern for educational group, with participants completing high school education more likely to report current smoking $(\mathrm{OR}=1.97$; $95 \%$ CI 1.41 to 2.74$)$. Stratified analysis by gender confirmed a declining prevalence of current smoking by age group for men and women (results not shown). Rural residence was a significant predictor of current smoking only for men (OR=1.41; 95\% CI 1.03 to 1.92). The prevalence of current smoking increased with level of education; however, this trend was stronger among women (results not shown). The $\chi^{2}$ test for trend has shown significant differences of current smoking prevalence across the age and education subgroups $(p<0.001$; results not shown).

\section{Univariate and multivariate analysis of risk factors for secondhand smoking}

Univariate analysis of secondhand smoking (table 3) indicated that male sex, age group (exposure to secondhand smoke declined with increasing age) and education level were significantly associated with secondhand smoke. Multivariate analysis confirmed male gender and age group were independent determinants of secondhand smoking (table 4).

\section{DISCUSSION}

The 2013 Greece GATS study enabled us to provide for the first time valuable and valid information about tobacco smoking patterns in Greece and its determinants. Our results found a high prevalence $(38.2 \%)$ of current smoking among adults in Greece, while previous nationwide studies found that the prevalence of smoking was estimated at $42.6 \%, 41 \%$ and $38.1 \%$ in 2008, 2010 and 2011, respectively. ${ }^{6}{ }^{7}$ However, there are considerable differences between these studies and the GATS in terms of methodology and overall response rate. A slightly higher $(39.3 \%)$ smoking prevalence has been found in the Russian Federation (GATS 2009). ${ }^{18} 19$ Among the European Union countries that released GATS results, Poland (30.5\%) and Romania (26.7\%) had considerably lower smoking rates compared with Greece. ${ }^{18-20}$ In addition, results from GATS from two South Eastern Mediterranean Region countries, Egypt $(19.7 \%)$ and Turkey (GATS 2012; 27.1\%), ${ }^{18-20}$ also showed lower smoking rates. Regarding determinants of current smoking, our results showed that sex was a strong independent determinant of current smoking. In particular, men had a higher adjusted OR than women. This result is higher in comparison to Poland's GATS results (2.4) but lower in comparison to GATS results from Egypt (162.2), Ukraine (13.1), Russian Federation (8.3) and Turkey (8.0). However, the prevalence of current smoking in Greece among women $(25.7 \%)$ is by 
Table 4 Multivariate analysis of current smoking and SHS (GATS, Greece, 2013)

\begin{tabular}{|c|c|c|c|c|c|c|}
\hline \multirow[b]{2}{*}{ Variable } & \multicolumn{3}{|c|}{ Current smoking } & \multicolumn{3}{|c|}{ SHS for non-smokers } \\
\hline & OR & $95 \% \mathrm{Cl}$ & p Value & $\overline{\text { OR }}$ & $95 \% \mathrm{Cl}$ & p Value \\
\hline \multicolumn{7}{|l|}{ Gender } \\
\hline Male & 3.24 & 2.62 to 4.00 & $<0.001$ & 2.12 & 1.45 to 3.10 & $<0.001$ \\
\hline Female & ref & & & ref & & \\
\hline \multicolumn{7}{|l|}{ Age } \\
\hline $25-39$ & 4.49 & 3.19 to 6.30 & $<0.001$ & 5.11 & 3.09 to 8.46 & $<0.001$ \\
\hline $40-54$ & 3.51 & 2.50 to 4.92 & $<0.001$ & 3.32 & 1.88 to 5.87 & $<0.001$ \\
\hline $55-64$ & 2.75 & 2.02 to 3.76 & $<0.001$ & 2.46 & 1.57 to 3.87 & $<0.001$ \\
\hline $65+$ & ref & & & ref & & \\
\hline \multicolumn{7}{|l|}{ Residence } \\
\hline Rural & 1.13 & 0.94 to 1.36 & 0.200 & 1.61 & 0.98 to 2.64 & 0.060 \\
\hline Urban & ref & & & ref & & \\
\hline \multicolumn{7}{|l|}{ Education level ${ }^{\star}, \dagger$} \\
\hline College or above & 1.67 & 1.10 to 2.51 & 0.016 & 0.92 & 0.53 to 1.60 & 0.773 \\
\hline High school & 1.97 & 1.41 to 2.74 & $<0.001$ & 1.04 & 0.64 to 1.67 & 0.876 \\
\hline Secondary & 1.76 & 1.21 to 2.58 & 0.004 & 1.20 & 0.78 to 1.84 & 0.403 \\
\hline Primary or less & ref & & & ref & & \\
\hline
\end{tabular}

*Primary or less includes 'no formal schooling', 'less than primary school completed' and 'primary school completed'; secondary includes 'less than secondary school completed', 'secondary school completed' and 'less than high school completed'; high school includes 'high school completed'; college or above includes 'college/university completed or technological educational institute' and 'postgraduate degree completed'.

†Education level is reported only among respondents $25+$ years old.

GATS, Global Adult Tobacco Survey; SHS, secondhand smoke.

far the highest in comparison to European countries (Poland 24.4\%, Romania 16.7\%, Russian Federation 21.7\%, Ukraine 11.2\%) and countries of South Eastern Mediterranean Region (Egypt 0.6\%, Turkey 13.1\%). Age was found to be a significant determinant of current smoking among adults in Greece. The prevalence of current smoking was considerably high $(30.0 \%)$ in the lowest age group. Taking into account the GATS data from the previous GATS study it is of note that only the Russian Federation (43.1\%) and Ukraine (30.5\%) recorded higher current smoking prevalence at the 1524 years old age group in comparison to Greece. Further, the prevalence of current smoking remained high in the age groups 25-34 (52.4\%) and 45-65 $(43 \%)$, while it decreased relatively steeply among participants of age $>65$ years. This age-related pattern of current smoking is similar to that observed (GATS data) in Poland, Romania, Russian Federation, Ukraine and Turkey. An interesting finding in the present study is the considerably high prevalence $(43 \%)$ of current smoking in the participants aged 45-64 years which is in line with the findings of the first GATS study conducted among 14 countries. This finding is of importance given that the health hazards of tobacco use begin to appear when adults reach middle age. On the basis of the above targeting cessation in these age groups is of crucial importance. Regarding the place of residence, we found similar prevalence of current smoking between urban and rural areas. The first GATS study revealed heterogeneous results across various countries. In particular, significantly higher prevalence of current smoking in urban areas in comparison to rural areas has been observed in eight countries: Egypt, Mexico, Poland, Russian Federation, Ukraine, Uruguay and Vietnam. On the contrary in two countries (India and Thailand) a statistically significantly increased prevalence of current smoking has been found in rural areas in comparison to the urban place of residence. ${ }^{21}$ Regarding residence status, we found no association between residence and current or secondhand smoking.

An interesting finding of our study was the positive association of education with current smoking prevalence. This finding indicates a pattern of smoking in Greece which is in contrast to the patterns of smoking in the West. It is well known that in the Western social context smoking is more prevalent among individuals with lower education. ${ }^{22}$ In Western countries individuals with higher education level may have better knowledge about the health hazards of smoking. In addition, persons with increasing education are getting more benefits from their participation in health promotion activities than persons with less education. ${ }^{23}$ The high prevalence of smoking and the positive association between educational level and current smoking reflect the low level of antismoking campaign in Greece. If a systematic antismoking health promotion campaign were to be implemented in Greece, we would expect a lower overall prevalence of current smoking, especially in the higher educational classes. Remarkably, the implementation of a systematic antismoking campaign in Greece in 1978 resulted in a substantial decrease in overall smoking rates. When the campaign stopped the smoking rate increased steadily to precampaign levels. $^{23}{ }^{24}$ Finally, the finding that almost $78 \%$ of ever 
daily smokers initiated daily smoking at the age 15-19 years underlines the need for tailored antismoking interventions targeting these age groups or even in younger age.

In 2010, a comprehensive smoke-free law was adopted by the Greek Parliament (in line with the Council Recommendation 2009/C 296/02 and with Article 8 of WHO Framework Convention on Tobacco Control) mandating a complete ban in all closed private and public places (Law 3868/2010). Nevertheless, our study indicates that the existing smoking bans for workplaces and other public places have not been satisfactorily implemented and enforced. Moreover, the fact that $90 \%$ of the Greek population is exposed to tobacco smoke (current smoking and secondhand smoke) suggests that smoking is a poorly controlled hazard despite the fact that since 2009 a considerable number of national tobacco control legislations have been enacted. ${ }^{25}$

Our study has some strengths and limitations. It is an advantage of our study that we used a representative sample of the adult Greek population and sampling and completion of the study were based on a standardised protocol to ensure international comparability across countries. In addition, the satisfactory response rate $(69 \%)$ is a strength of our study indicating a good representativeness of the general population, and a similar rate has been observed in other GATS studies. ${ }^{26}$ Further, the data have been collected by trained interviewers and this could have reduced the inter-reviewer error. The limitations of our study are mainly related to the selfreported nature of the data collected. Self-reported data may lead to underestimation of smoking prevalence. ${ }^{18}$ Another limitation is related to the cross-sectional design of the GATS. ${ }^{26-27}$ In addition, we were not able to obtain data from the non-responders. However, these limitations are counterbalanced by the methodological advantages previously mentioned.

In conclusion, the first GATS study in Greece revealed a high prevalence of current smoking and exposure to secondhand smoke among the adult population and also a positive social gradient between education and current smoking. Smoking is an uncontrolled hazard in Greece. These findings are alarming and implementation of comprehensive tobacco control and prevention strategies could be impactful in fighting the tobacco epidemic in Greece.

\author{
Author affiliations \\ ${ }^{1}$ Faculty of Medicine, Department of Hygiene and Epidemiology, University of \\ Thessaly, Larissa, Greece \\ ${ }^{2}$ Department of Public and Administrative Health, National School of Public \\ Health, Athens, Greece \\ ${ }^{3}$ Regional Office for Europe, World Health Organization, Copenhagen, \\ Denmark
}

Acknowledgements We would like to thank the Global Tobacco Control Branch, Office on Smoking and Health, National Center for Chronic Disease Prevention and Health Promotion, Centers for Disease Control and Prevention, Atlanta, Georgia, USA for the support in designing and implementing the study. Moreover, from the same Department, we would like to specifically acknowledge the contribution and support of Jeremy Morton.

Contributors GR participated in study design, drafted and revised the manuscript. $A B$ participated in study design, supervised the study implementation, drafted and revised the manuscript. AK participated in study design and statistical analysis. EA, KK and KM participated in study design and revised the manuscript. KKour participated in data collection and revision of the manuscript. JM, AT and JK participated in study design and revised the manuscript for important intellectual content. RNK participated in study design. $\mathrm{CH}$ supervised study design and the implementation of the study, participated in statistical analysis, interpretation of results and revision of the manuscript. All authors have read and approved the final version of the manuscript.

Funding The GATS Greece 2013 is implemented through the Operational Programme entitled 'Human Resources Development' of National Strategic Reference Framework (NSRF) 2007-2013 (number agreement IMS-328/773). The programme is co-funded by Greece and the European Union-European Regional Development Fund. Chronic Disease Prevention and Health Promotion, Centers for Disease Control and Prevention, USA (CDC) provided technical assistance.

Competing interests None declared.

Patient consent Obtained.

Ethics approval Approval of the study protocol was received by the Institutional Review Board of National School of Public Health (NSPH), Athens, Greece.

Provenance and peer review Not commissioned; externally peer reviewed.

Data sharing statement The data for Greece GATS 2013 are available from CDC (http://nccd.cdc.gov/GTSSData/Ancillary/DataReports.aspx?CAID=2)

Open Access This is an Open Access article distributed in accordance with the Creative Commons Attribution Non Commercial (CC BY-NC 4.0) license, which permits others to distribute, remix, adapt, build upon this work noncommercially, and license their derivative works on different terms, provided the original work is properly cited and the use is non-commercial. See: http:// creativecommons.org/licenses/by-nc/4.0/

\section{REFERENCES}

1. World Health Organization (WHO). Global health risks-mortality and burden of disease attributable to selected major risks. 2009. http://www.who.int/healthinfo/global burden disease/ GlobalHealthRisks_report_full.pdf (accessed 12 Apr 2016).

2. World Health Organization (WHO). WHO report on the global tobacco epidemic, 2011: warning about the dangers of tobacco. 2011. http://apps.who.int/iris/bitstream/10665/44616/1/ 9789240687813 eng.pdf (accessed 12 Apr 2016).

3. European Commission. Agriculture and Rural Development, Raw Tobacco. http://ec.europa.eu/agriculture/tobacco/index_en.htm (accessed 12 Apr 2016).

4. Harvard School of Public Health. The Greek Tobacco Epidemic. 2011. http://www.who.int/fctc/reporting/party_reports/greece annex1_the_greek_tobacco_epidemic_2011.pdf (accessed 12 Apr 2016).

5. World Health Organization. World Health Statistics 2011. 2011. http:/ www.who.int/whosis/whostat/EN_WHS2011_Full.pdf?ua=1 (accessed 12 Apr 2016).

6. Filippidis FT, Vardavas $\mathrm{Cl}$, Loukopoulou A, et al. Prevalence and determinants of tobacco use among adults in Greece: 4 year trends. Eur J Public Health 2013;23:772-6.

7. Filippidis FT, Schoretsaniti S, Dimitrakaki C, et al. Trends in cardiovascular risk factors in Greece before and during the financial crisis: the impact of social disparities. Eur J Public Health 2014;24:974-9.

8. Filippidis FT, Tzavara Ch, Dimitrakaki C, et al. Compliance with a healthy lifestyle in a representative sample of the Greek population: preliminary results of the Hellas Health I study. Public Health 2011;125:436-41.

9. Rachiotis G, Behrakis PK, Vasiliou M, et al. Quality of life and smoking among industrial workers in Greece. Med Lav 2006:97:44-50. 
10. Drivas S, Rachiotis G, Vlastos FD, et al. Occupational exposure to lignite and impact on respiratory system among heavy industry personnel. Ind Health 2007;45:409-14.

11. Rachiotis G, Karydis I, Drivas S, et al. Pattern of smoking habit among Greek blue and white collar workers. Int J Environ Res Public Health 2009;6:1812-17.

12. World Health Organization (WHO). WHO report on the Global Tobacco Epidemics, 2008. The MPOWER Package, 2008. http:// www.who.int/tobacco/mpower/mpower_report_full_2008.pdf (accessed 12 Apr 2016)

13. Asma S, Song Y, Cohen J, et al. CDC Grand Rounds: global tobacco control. MMWR Morb Mortal Wkly Rep 2014;63:277-80.

14. Global Adult Tobacco Survey Collaborative Group. Global Adult Tobacco Survey (GATS): Sample Design Manual, version 2.0. Atlanta, GA: Centers for Disease Control and Prevention, 2010. http://www.who.int/tobacco/surveillance/en_tfi_gats_sample designmanual_v2_final_03nov2010.pdf (accessed 12 Apr 2016).

15. Global Adult Tobacco Survey Collaborative Group. Global Adult Tobacco Survey (GATS): Sample Weights Manual, version 2.0. Atlanta, GA: Centers for Disease Control and Prevention, 2010. http://who.int/tobacco/surveillance/en_tfi_gats_sampleweights manual_v2_final_15dec2010.pdf (accessed 12 Apr 2016).

16. Global Adult Tobacco Survey. Country Report, Greece 2013. http:/ www.who.int/tobacco/surveillance/survey/gats/grc_country_report.pdf (accessed 12 Apr 2016)

17. Barbouni A, Rachiotis G, Kremastinou T. Data concerning tobacco use in Greece. HCDCP, E-bulletin, 2014. http://www2.keelpno.gr/ blog/?p=5542\&lang=en (accessed 12 Apr 2016).
18. Song Y, Zhao L, Palipudi KM, et al. Tracking MPOWER in 14 countries: results from the Global Adult Tobacco Survey, 20082010. Glob Health Promot 2016;23(Suppl):24-37.

19. Kostova D, Andes L, Erguder T, et al. Cigarette prices and smoking prevalence after a tobacco tax increase-Turkey, 2008 and 2012 MMWR Morb Mortal Wkly Rep 2014;63:457-61.

20. Giovino GA, Mirza SA, Samet JM, et al. Tobacco use in 3 billion individuals from 16 countries: an analysis of nationally representative cross-sectional household surveys. Lancet 2012;380:668-79.

21. Palipudi KM, Gupta PC, Sinha DN, et al. Social determinants of health and tobacco use in thirteen low and middle income countries: evidence from Global Adult Tobacco Survey. PLoS ONE 2012;7: e33466.

22. Cavelaars AE, Kunst AE, Geurts JJ, et al. Educational differences in smoking: international comparison. BMJ 2000;320:1102-7.

23. Doxiadis SA, Trihopoulos DV, Phylactou HD. Impact of a nationwide anti-smoking campaign. Lancet 1985;2:712-13.

24. Vardavas $\mathrm{Cl}$, Kafatos A. Greece's tobacco policy: another myth? Lancet 2006;367:1485-6.

25. Vardavas $\mathrm{Cl}$, Behrakis P. Greece: action at last! Tob Control 2009:18:79-80.

26. Kaleta D, Usidame B, Biliński $P$, et al. Global Adult Tobacco Survey (GATS) in Poland 2009-2010—study strengths, limitations and lessons learned. Ann Agric Environ Med 2012;19:717-22.

27. Kaleta D, Makowiec-Dabrowska T, Dziankowska-Zaborszczyk E, et al. Predictors of smoking initiation-results from the Global Adult Tobacco Survey (GATS) in Poland 2009-2010. Ann Agric Environ Med 2013;20:756-66. 